HD-THEP-00-10

\title{
Thermal Quantum Fields in Static Electromagnetic Backgrounds
}

\author{
A.A. Actor \\ Department of Physics, The Pennsylvania State University \\ Lehigh Valley Campus, Fogelsville, PA 18051, U.S.A. 用 \\ K. D. Rothe \\ Institut für Theoretische Physik - Universität Heidelberg \\ Philosophenweg 16, D-69120 Heidelberg
}

\begin{abstract}
We present and discuss, at a general level, new mathematical results on the spatial nonuniformity of thermal quantum fields coupled minimally to static background electromagnetic potentials. Two distinct examples are worked through in some detail: uniform (parallel and perpendicular) background electric and magnetic fields coupled to a thermal quantum scalar field.
\end{abstract}

*e-mail: aaa2@psu.edu

$\dagger$ e-mail: k.rothe@thpys.uni-heidelberg.de 


\section{Introduction and General Results}

In thermal quantum field theory (hereafter thermal QFT) with matter fields coupled to an external gauge potential it has long been known [1]-[7] that a constant Euclidean gauge potential $A_{0}^{E}=a=$ constant is a compact physical parameter. Upon first encounter this may seem strange, because at zero temperature $(T=0)$ one can simply remove such a constant by means of a gauge transformation $A_{\mu}^{E} \rightarrow A_{\mu}^{E}-\partial_{\mu} \lambda$ with $\lambda=x_{0} A_{0}^{E}$. However, in the Matsubara or imaginary time formalism [8, 9] (which we use throughout this paper) bosonic (fermionic) matter fields must be periodic (antiperiodic) functions of (Euclidean) time, with period $\beta=\frac{1}{T}$. This restricts the allowed gauge transformations to those satisfying $\lambda\left(x_{0}+\beta\right)=\lambda\left(x_{0}\right)+\eta \pi \bmod 2 \pi$, with $\eta=0$ [1] for bosonic (fermionic) matter fields. Hence the constant $a$ can only be removed if $a=(2 N+\eta)(\pi / \beta)$.

It follows from above that it is always possible to gauge an arbitrary $A_{0}^{E}(\vec{x}) \underline{\text { into }}$ the physical interval $0 \leq A_{0}^{E}(\vec{x}) \leq \frac{2 \pi}{\beta}$ at every point $\vec{x}$. Moreover, $\beta A_{0}^{E}=2 \pi$ is gauge-equivalent to $\beta A_{0}^{E}=0$; hence the real dimensionless quantity $\beta A_{0}^{E}$ is compact - an angle. What physics underlies this angular variable? A specific and complete answer to this question can be given. Though the essential facts have been known for a long time, many in the field theory community seem not to be aware of this physical picture, as we have not found it discussed in the literature.

The physical meaning of the angle $\beta A_{0}^{E}$ emerges from the old observation $\beta$

†Throughout this paper we shall move freely back and forth between Euclidean and Minkowski spacetime. For sake of clarity we attach the label E to (certain) Euclidean quantities, and in particular to the Euclidean gauge potential.

$\S$ Note that we absorb the unit $e$ of electric charge carried by the particles of the matter field into the gauge potential $A_{\mu}^{E}$. Thus the covariant derivative is $D_{\mu}=\partial_{\mu}-i A_{\mu}^{E}$, and $A_{\mu}^{E}$ has, in natural units, the dimension of energy. In particular, $A_{0}^{E}(\vec{x})$ is the electrostatic energy of a positive charge at point $\vec{x}$. To discuss voltage explicitly, we refer to $A_{0}^{E}(\vec{x}) / e$. 
(see e.g. refs. [10, 11]) that an imaginary constant Euclidean gauge potential

$$
A_{0}^{E}=i A_{0}, \quad A_{0}=\mu
$$

corresponds to a chemical potential $\mu$ for the thermal matter field to which $A_{\mu}$ is minimally coupled. This is true for both spinor and scalar thermal fields. Eq. (1.1) is written to emphasize that, eventually, one must change from the background Euclidean potential $A_{\mu}^{E}=\left(A_{0}^{E}, \vec{A}\right)$ to the corresponding background Minkowski potential $A_{\mu}=\left(-i A_{0}^{E}, \vec{A}^{E}\right)$ when writing down one's final physical formulae. The identification $A_{0}=\mu$ as a chemical potential is merely the recognition that for a charged particle in a uniform background voltage $A_{0} / e$ pervading all of space, $A_{0}$ has the meaning of a chemical potential: it is the electrostatic energy that must be expended to create the charged particle at whatever position $\vec{x}$ this particle occupies. In sect. 2 we similarly recognize that a nonuniform background voltage $A_{0}(\vec{x}) / e$ has locally this same interpretation.

The physical significance of a constant $A_{0}^{E} / e$ is now clear. It represents, in Euclidean language, a uniform voltage $A_{0} / e$ throughout space. Clearly $A_{0}=$ constant is a true physical parameter - noncompact obviously - a voltage which is felt by any real charged particle, and felt in particular by the real particles of the thermal plasma. In the limit $T \rightarrow 0$ this plasma disappears, leaving the virtual particle sea, which is not sensitive to a uniform voltage throughout space. The sea knows nothing about uniform background voltage because the virtual pairs of which the sea consists have precisely zero electrostatic energy in such a background.

Once it is known that Euclidean $\beta A_{0}^{E}$ is an angular variable one can, using the power of Fourier analysis, write all gauge-invariant physical functions as Fourier cosine series in this angle. Thus for, respectively, the diagonal elements of the Euclidean heat kernel $h^{(\beta)}$, effective Lagrangian $\mathcal{L}^{(\beta)}$ and 
stress tensor $T_{\mu \nu}^{(\beta)}$ of the thermal quantum field we can write

$$
\begin{gathered}
h^{(\beta)}(t \mid \vec{x}, \vec{x})=\sum_{n=0}^{\infty}( \pm)^{n} h_{n}^{(\beta)}(t \mid \vec{x}) \cos \left(n \beta A_{0}^{E}(\vec{x})\right), \\
\mathcal{L}^{(\beta)}(\vec{x})=\sum_{n=0}^{\infty}( \pm)^{n} \mathcal{L}_{n}^{(\beta)}(\vec{x}) \cos \left(n \beta A_{0}^{E}(\vec{x})\right), \\
T_{\mu \nu}^{(\beta)}(\vec{x})=\sum_{n=0}^{\infty}( \pm)^{n} T_{\mu \nu ; n}^{(\beta)}(\vec{x}) \cos \left(n \beta A_{0}^{E}(\vec{x})\right) .
\end{gathered}
$$

Here in each formula the coefficients of $\cos \left(n \beta A_{0}^{E}(\vec{x})\right)$ depend on $\overrightarrow{\mathcal{E}}(\vec{x})=$ $-\vec{\nabla} A_{0}^{E}(\vec{x})$ and $\vec{B}(\vec{x})=\vec{\nabla} \times \vec{A}(\vec{x})$, but not directly on $A_{0}^{E}(\vec{x})$. Our use of $A_{0}^{E}(\vec{x})$ here implies an arbitrary static Euclidean gauge potential, and that will be our final result. Sect. 2 is devoted to demonstrating the above implied compactification of $A_{0}^{E}(\vec{x})$ at the local level. The nonalternating/alternating sign $( \pm)^{n}$ is appropriate for scalar/spinor thermal matter fields. One could equivalently disregard this sign and replace $A_{0}^{E}$ by $A_{0}^{E}-\eta(\pi / \beta)$ everywhere with $\eta=0$ [1] for scalar (spinor) field.

It is worth pointing out that eqs. (1.2)-(1.4) display the expected complete separation of all functions characterizing the thermal field into parts representing the virtual sea and thermal plasma. E. g. for the heat kernel we have

$$
h^{(\beta)}=h_{\text {sea }}+h_{\text {plasma }}^{(\beta)}
$$

where

$$
\begin{gathered}
h_{\text {sea }}=h_{0}(t \mid \vec{x}), \\
h_{\text {plasma }}^{(\beta)}=\sum_{n=1}^{\infty}( \pm)^{n} h_{n}^{(\beta)}(t \mid \vec{x}) \cos \left(n \beta A_{0}^{E}(\vec{x})\right) .
\end{gathered}
$$

where $h_{0}(t \mid \vec{x})$ is the $T=0$ heat kernel and represents the vacuum or virtual particle sea contribution to the thermal heat kernel. The vacuum is always independent of $T$ : its virtual particles do not have the prolonged existence needed to come into thermal equilibrium with anything. Neither does the vacuum feel directly an applied voltage, so $h_{\text {sea }}$ cannot depend explicitly on $A_{0}^{E}$. Similarly $\mathcal{L}^{(\beta)}$ and $T_{\mu \nu}^{(\beta)}$ separate into sea $(n=0)$ and plasma $(n>0)$ 
parts, the former being independent of $\beta$ and $A_{0}^{E}$, while the latter depend on both $\beta$ and $A_{0}^{E}$.

Before embarking on calculations, a few words about background $\overrightarrow{\mathcal{E}}$ and $\vec{B}$ fields interacting with the vacuum and with the thermal plasma may be of use to some readers.

\section{Virtual sea}

The standard visualization of vacuum quantum fluctuations as virtual pairs - initially zero-length "vacuum dipoles" which grow to maximum size, then shrink again to zero length and annihilate away - enables one also to visualize the effect of a background $A_{0}$, as well as the effect of electric and magnetic fields, on these fluctuations. Due to their mutual "binding", virtual pairs do not feel $A_{0}(\vec{x})$ directly. This was already mentioned for constant $A_{0}$. The vacuum pair however couples to any nonuniformity in $A_{0}(\vec{x})$ - i.e. to the electric field.

A background electric field exerts an aligning torque on vacuum dipoles - the famous "vacuum polarization" effect. $\vec{E}$ also tries to stretch or shorten these nonrigid dipoles, depending on their orientation relative to $\vec{E}$. A vacuum dipole whose moment is parallel to $\vec{E}$ will be stretched and, perhaps, even given enough external energy to break apart into real particles. Dipoles antiparallel to $\vec{E}$ will be shortened; those perpendicular to $\vec{E}$ only rotated. Real pair creation occurs from the vacuum, preferentially along the direction of $\vec{E}$, but not perpendicular to $\vec{E}$. Pair creation is independent of $T$. As in Schwinger's original $T=0$ calculation [12 and the subsequent literature known to us (see e.g. the books [13]-15]), our calculations predict the phenomenon of pair creation, but do not take account of these pairs once they have been produced. All such calculations treat pair creation as a perturbation of a pre-existing many-body system - the virtual sea or the sea plus plasma, with fixed background $\vec{E}$ and $\vec{B}$ - whose subsequent development is 
not investigated.

A background $\vec{B}$ cannot transfer energy to individual particles, and therefore cannot cause particle production from the vacuum. Evidently, $\vec{B}$ acts locally (more or less rigidly) to displace vacuum pairs, but not to stretch or shorten them. To this limited extent the vacuum can be aware of $\vec{B}$.

\section{Thermal plasma}

The thermal plasma is a neutral quantum gas of unbound charged real particles. Pair creation does not occur from the thermal plasma. Through their electrostatic energy these particles know individually about the background potential $A_{0}(\vec{x})$ - our main point leading to eqs.(1.2)-(1.4). If $\vec{E}(\vec{x})=$

$-\vec{\nabla} A_{0}(\vec{x}) \neq 0$, these particles feel individually the Coulomb force $\vec{F}=q \vec{E}$, which of course accelerates $q=e(-e)$ parallel (antiparallel) to $\vec{E}$. Neither our calculation, nor others in the $T>0$ literature follow up the consequences of this acceleration. Through their thermal motion, the plasma particles also feel the magnetic force $\vec{F}=q \vec{v} \times \vec{B}$ perpendicular to $\vec{B}$. Functions describing the thermal plasma therefore depend on $A_{0}(\vec{x}), \vec{E}$ and $\vec{B}$.

\section{Euclidean Spacetime}

\footnotetext{
ॠFor sake of completeness we mention here the non-equilibrium approach to background fields 16. In this approach the existence of an electric field $\vec{E}_{0}$ is assumed as initial condition with, say, no real pairs present. Pair production ensues, and the produced pairs serve in turn as sources of an additional electric field: charge separation occurs, first partially, cancelling, then strongly overcancelling $\vec{E}_{0}$. More pairs are produced and things go in reverse. Eventually plasma oscillations set in. These calculations involve large sets of coupled equations and are intensively numerical. There is no thermal equilibrium, and hence no temperature. Our analytic work here could serve as the $T>0$ initial conditions for such numerical investigations.
} 


\subsection{Compactification}

The Fourier series (1.2)-(1.4) rest upon the fact that in any gauge-invariant quantity, $\beta A_{0}^{E}(\vec{x})$ plays the role of a local compact angle, $0 \leq \beta A_{0}^{E}(\vec{x}) \leq 2 \pi$, since, as we have seen, one can gauge this function into the interval $\left[0, \frac{2 \pi}{\beta}\right]$ at any point $\vec{x}$ in space. Moreover, the upper and lower ends of this interval can be identified by a gauge transformation. Note that this compactification does not extend to a time-dependent gauge potential $A_{0}^{E}$. Indeed, within the Matsubara formalism one cannot accommodate time-dependent backgrounds of any kind.

The Fourier series (1.2)-(1.4) express, or are the result of, a remarkable series resummation, as we shall illustrate in subsection 2.3 below. Let us briefly recall the mode-sum construction of the thermal heat kernel for a scalar field coupled to an arbitrary static background potential $A_{\mu}(\vec{x})$ [17]. The spacetime Matsubara modes

$$
\phi_{m p}\left(x_{0}, \vec{x}\right)=\frac{1}{\sqrt{\beta}} e^{i(2 \pi m / \beta) x_{0}} \varphi_{m p}(\vec{x})
$$

satisfy $\left(-D^{2}\right) \phi_{m p}=\lambda_{m p}^{2} \phi_{m p}$, where $D_{\mu}=\partial_{\mu}-i A_{\mu}^{E}, m$ runs over all integers, and $p$ is a collective label for all spatial directions. The modes $\varphi_{m p}(\vec{x})$ satisfy

$$
\left[\left(A_{0}^{E}(\vec{x})-\frac{2 \pi m}{\beta}\right)^{2}-(\vec{\nabla}-i \vec{A}(\vec{x}))^{2}\right] \varphi_{m p}(\vec{x})=\lambda_{m p}^{2} \varphi_{m p}(\vec{x}) .
$$

Here the $\left(A_{0}^{E}-2 \pi m / \beta\right)^{2}$ term in the bracket has particular importance. It couples position $\vec{x}$ to the Matsubara label $m$. Also, it displays the local compactification of $A_{0}^{E}$ : the shift $A_{0}^{E} \rightarrow A_{0}^{E}-2 \pi N / \beta$ merely shifts the Matsubara label, $m \rightarrow m+N$.

The thermal heat kernel of the operator $-D_{\mu}^{2}$ is defined by

$$
h^{(\beta)}(t \mid x, y)=\sum_{m, p} e^{-t \lambda_{m p}^{2}} \phi_{m p}(x) \phi_{m p}^{*}(y) .
$$

The corresponding diagonal elements

$$
\left.h^{(\beta)} t \mid x, x\right)=\sum_{m, p} e^{-t \lambda_{m p}^{2}}\left|\varphi_{m p}(\vec{x})\right|^{2}
$$




$$
=\sum_{n} h_{n}^{(\beta)}(t \mid \vec{x}) \cos \left(n \beta A_{0}^{E}(\vec{x})\right)
$$

display the mode sum resummation to a Fourier series, alluded to above. For reasons of gauge invariance the coefficients in eq. (2.4) can only depend on the Euclidean electric field $-\vec{\nabla} A_{0}^{E}$ and magnetic field $\vec{B}=\vec{\nabla} \times \vec{A}$, but not on the potential $A_{0}^{E}$ directly. Except for the $n=0$ coefficient, they also depend

on the temperature. In the limit $T \rightarrow 0$ all the $h_{n \neq 0}^{(\beta)}$ vanish exponentially (the thermal plasma disappears), and what remains is the $T=0$ heat kernel (1.6) for the virtual sea. All of these statements are illustrated by the example in subsection 2.3 below, and those in sections 4 and 5 .

\subsection{Effective Lagrangians}

Much of the early work on thermal quantum fields coupled to background gauge fields was concerned with effective Lagrangians for the potential $A_{0}^{E}$ (see e.g. refs. [2], [6], [7]). A related theme was the study of "order parameters" which signaled the deconfinement phase transition at high $T$ in nonabelian gauge theories (see e.g. refs. [3, 困, 5]). Our formula (1.3) has a natural interpretation as an effective Lagrangian $=\mathcal{L}^{(\beta)}$. The coefficients $\mathcal{L}_{n}^{(\beta)}(\vec{x})$ of $\cos \left(n \beta A_{0}^{E}\right)$ in eq. (11.3) are actually functions of $\overrightarrow{\mathcal{E}} \cdot \overrightarrow{\mathcal{E}}=\left(\vec{\nabla} A_{0}^{E}\right)^{2}$ (not to mention $\vec{B} \cdot \vec{B}$ which we suppress here), and therefore play the role of (very complicated) "kinetic terms" in $\mathcal{L}^{(\beta)}\left(A_{0}^{E}\right)$. The $\cos \left(n \beta A_{0}^{E}\right)$ factors play the role of "potential terms" in the same Lagrangian. An expansion of $\mathcal{L}^{(\beta)}$ in powers of $\overrightarrow{\mathcal{E}} \cdot \overrightarrow{\mathcal{E}}$ and $\left(A_{0}^{E}\right)^{2}$ has the form

$$
\begin{aligned}
\mathcal{L}_{\beta}= & a_{0}+a_{1}\left(A_{0}^{E}\right)^{2}+\cdots \\
& +\left(\vec{\nabla} A_{0}^{E}\right)^{2}\left[b_{0}+b_{1}\left(A_{0}^{E}\right)^{2}+\cdots\right] \\
& +\cdots
\end{aligned}
$$

where we find the conventional kinetic term among all the others. Here the coefficients $a_{n}, b_{n}, \ldots$ are independent of $A_{0}^{E}$ and $\overrightarrow{\mathcal{E}}$ (but depend on background $\vec{B})$. 


\subsection{Fourier series and resummation}

In the following we wish to illustrate, for the case of fermions in one space dimension, how the Fourier series in (1.1) is the result of an infinite resummation of the Matsubara sum. Thermal fermionic fields must, of course, satisfy antiperiodic boundary condition in $x_{0}$. Let us consider eqs. (2.1), (2.2) for a scalar field satisfying the antiperiodic boundary condition $\phi\left(x_{0}+\beta\right)=-\phi\left(x_{0}\right)$ in Euclidean time. This only requires the replacement $m \rightarrow m+1 / 2$ in eqs. (2.1), (2.2). In the mode equation (2.2) the $1 / 2$ can be absorbed into the gauge potential, $A_{0}^{E} \rightarrow A_{0}^{E}-\pi / \beta$, leaving everything else just as it was. Consequently, the only change in the heat kernel (2.4) and related Fourier series is

$$
\cos \left(n \beta A_{0}^{E}\right) \rightarrow(-)^{n} \cos \left(n \beta A_{0}^{E}\right)
$$

The preceding argument indicates that for thermal Fermi fields one will have the alternating signs displayed in eqs. (1.2)-(1.4).

As is well known (see e.g. ref. [18, 27] and references therein), the small $t$ expansion of heat kernels is of the form

$$
h^{(\beta)}(t ; x, x) \sim \sum_{k=0}^{\infty} t^{(k-d-1) / 2} a_{k}^{(\beta)}(\vec{x}), t \rightarrow 0
$$

where $d$ is the space-time dimension, and where the coefficients $a_{k}^{(\beta)}(\vec{x})$ depend on the quantum field as well as the structure of space-time in which the quantum field lives. Given our knowledge of the Fourier series (2.4), we can make the obvious prediction

$$
a_{k}^{(\beta)}(\vec{x})=\sum_{n}( \pm)^{n} a_{k n}^{(\beta)}(\vec{x}) \cos \left(n \beta A_{0}^{E}(\vec{x})\right) .
$$

This statement goes far beyond the standard lore of asymptotic heat kernel expansions. It is instructive to see how this periodicity comes about in the context of a Seeley expansion.

In 19 it was shown that in two space-time dimensions the heat kernel for the Dirac operator of massless fermions in an external, static gauge field 
$A_{0}^{E}=\left(\mathcal{E} x_{1}+\frac{2 \pi a}{\beta}, A_{1}=0\right)$ takes the form

$\operatorname{trh}^{(\beta)}(t ; x, x)=\frac{\mathcal{E}}{2 \pi}\left(\frac{1}{\tanh \mathcal{E} t}\right)\left\{1+2 \sum_{n=1}^{\infty}(-1)^{n} \cos \left[n \beta\left(\mathcal{E} x_{1}+\frac{2 \pi}{\beta} a\right)\right] e^{-\frac{n^{2} \beta^{2} \mathcal{E}}{4 \tanh \mathcal{E} t}}\right\}$,

where we see the anticipated alternating sign in the sum. Expanding the $\mathcal{E}$-dependent multiplicative factor as well as the exponential in powers of $t$,

$$
\begin{gathered}
\frac{\mathcal{E}}{2 \pi}\left(\frac{1}{\tanh \mathcal{E} t}\right)=1+\frac{1}{3}(\mathcal{E} t)^{2}+\cdots \\
e^{-\frac{n^{2} \beta^{2} \mathcal{E}}{4 \tanh \mathcal{E} t}}=\left(1-\frac{1}{12} n^{2} \beta^{2} \mathcal{E}^{2} t+\cdots\right) e^{-\frac{n^{2} \beta^{2}}{4 t}}
\end{gathered}
$$

one finds from (2.8) for the diagonal elements of the heat kernel,

$$
\begin{aligned}
2 \pi \operatorname{trh}^{(\beta)}(t ; x, x) & =\frac{1}{t}\left[1+2 \sum_{n=1}^{\infty}(-)^{n} e^{-\frac{n^{2} \beta^{2}}{4 t}} \cos \left[n\left(\mathcal{E} \beta x_{1}+2 \pi a\right]\right]\right. \\
& -\frac{1}{6} \sum_{n=1}^{\infty}(-)^{n} e^{-\frac{n^{2} \beta^{2}}{4 t}} n^{2} \beta^{2} \mathcal{E}^{2} \cos \left[n\left(\mathcal{E} \beta x_{1}+2 \pi a\right)\right]+O(t) .
\end{aligned}
$$

On the other hand it was shown in Ref. [26] (see also [19]) that the above heat kernel possesses a formal expansion of the form

$$
h^{(\beta)}(t ; x, x)=\frac{1}{4 \pi t} \sum_{\ell=0}^{\infty} a_{\ell}\left(x ; \frac{\sqrt{t}}{\beta}\right) t^{\ell}
$$

where

$$
\begin{aligned}
a_{\ell}\left(x ; \frac{\sqrt{t}}{\beta}\right) & =\sqrt{\frac{4 \pi t}{\beta^{2}}} \int \frac{d k_{1}}{\sqrt{\pi}} \sum_{m=-\infty}^{\infty} e^{-\left(k_{1}^{2}+\bar{\omega}_{m}^{2}\left(\frac{\sqrt{t}}{\beta}\right)\right)} \\
& \times\left\{\sum_{r=0}^{\ell} \sum_{\text {dist.perm. }} \frac{(-1)^{\ell-r}}{(\ell+r) !}(2 i k \cdot D)^{2 r} \hat{D}^{\ell-r}\right\}_{k_{2}=\bar{\omega}_{m}\left(\frac{\sqrt{t}}{\beta}\right)} \cdot \mathbf{1}
\end{aligned}
$$

where the sum is over all distinct permutations, $\bar{\omega}_{m}$ are the scaled Matsubara frequencies

$$
\bar{\omega}_{m}\left(\frac{\sqrt{t}}{\beta}\right)=2 \pi\left(m+\frac{1}{2}\right)\left(\frac{\sqrt{t}}{\beta}\right)=\sqrt{t} \omega_{m} .
$$

and

$$
D_{\mu}=\partial_{\mu}-i A_{\mu}^{E}, \quad \hat{D}=-\partial^{2}+\left(A_{0}^{E}\right)^{2}+X
$$

with $X$ a matrix valued field $\left(\epsilon_{01}=1\right)$

$$
X=-\frac{1}{2} \gamma^{5} \epsilon_{\mu \nu} \partial_{\mu} A_{\nu}^{E}
$$


In (2.11) we have already taken account of the fact, that only even powers in $k_{1}$ and $\bar{\omega}_{m}$ will contribute to the integral and sum in (2.11). The leading contribution to (2.11) for $t \rightarrow 0$ is given by the $r=\ell$ term in the sum, and in particular by the term $\bar{\omega}_{m} A_{0}^{E}$ in $i k \cdot D$. Hence,

$$
\begin{aligned}
a_{\ell}\left(x ; \frac{\sqrt{t}}{\beta}\right) & \approx \sqrt{\frac{t}{\beta^{2}}} \int \frac{d k_{1}}{\sqrt{\pi}} e^{-k_{1}^{2}} \\
& \times \sqrt{4 \pi} \frac{1}{(2 \ell) !} \sum_{m} e^{-\bar{\omega}_{m}^{2}}\left(4 \omega_{m}^{2}\right)^{\ell}\left(A_{0}^{E}\right)^{2 \ell} \\
& =\sqrt{\frac{t}{\beta^{2}}} \bar{I}_{\ell} \frac{\left(A_{0}^{E}\right)^{2 \ell}}{(2 \ell) !}+O\left(\frac{t}{t^{\ell}}\right) \quad(\ell>0) .
\end{aligned}
$$

where

$$
\bar{I}_{\ell}=\sqrt{4 \pi} \sum_{m=-\infty}^{\infty}\left(4 \bar{\omega}_{m}^{2}\right)^{\ell} e^{-\bar{\omega}_{m}^{2}}
$$

We obtain the expansion of $\bar{I}_{\ell}$ in powers of $t$ by repeatedly differentiating the Jacobi identity

$$
\sum_{m=-\infty}^{\infty} e^{-\tau\left[2 \pi\left(m+\frac{1}{2}\right)\right]^{2}}=\sqrt{\frac{1}{4 \pi \tau}}\left[1+2 \sum_{n=1}^{\infty}(-)^{n} e^{-\frac{n^{2}}{4 \tau}}\right] .
$$

with respect to $\tau$, and setting $\tau=\frac{t}{\beta^{2}}$. We thus find

$$
\begin{gathered}
\bar{I}_{0}=\sqrt{\frac{\beta^{2}}{t}}\left[1+2 \sum_{n=1}^{\infty}(-)^{n} e^{-\frac{n^{2} \beta^{2}}{4 t}}\right], \\
\bar{I}_{\ell}=\sqrt{\frac{\beta^{2}}{t}} 2(-1)^{\ell} \sum_{n=1}^{\infty}(-)^{n} e^{-\frac{n^{2} \beta^{2}}{4 t}}\left[\left(\frac{n^{2} \beta^{2}}{t}\right)^{\ell}+O\left(\frac{t}{t^{\ell}}\right)\right] .
\end{gathered}
$$

We thus finally have from (2.12)

$$
\begin{aligned}
a_{0}\left(x ; \frac{\sqrt{t}}{\beta}\right) & =\left[1+2 \sum_{n=1}^{\infty}(-)^{n} e^{-\frac{n^{2} \beta^{2}}{4 t}}\right] \\
t^{\ell} a_{\ell}\left(x ; \frac{\sqrt{t}}{\beta}\right) & =2(-)^{\ell} \sum_{n=1}^{\infty}(-)^{n} e^{-\frac{n^{2} \beta^{2}}{4 t}}\left[\frac{\left(n \beta A_{0}^{E}\right)^{2 \ell}}{(2 \ell) !}+O(t)\right] \quad(\ell>0) .
\end{aligned}
$$

Hence

$$
\sum_{\ell} t^{\ell} a_{\ell}\left(x ; \frac{\sqrt{t}}{\beta}\right)=1+2 \sum_{n=1}^{\infty}(-)^{n} e^{-\frac{n^{2} \beta^{2}}{4 t}}\left[\cos \left(n \beta A_{0}^{E}(x)\right)+O(t)\right] .
$$

Substitution of (2.16) into (2.10) reproduces the leading term in the small $t$ expansion (2.9) of the heat kernel. 
The corresponding calculation of next to leading order is very cumbersome due to the non-commutativity of the operators appearing in the expansion (2.11), and we shall not persue this any further.

\section{Minkowski Space-time}

Once we know eqs.(1.2)-(1.4) are valid for an arbitrary static Euclidean background potential $A_{0}^{E}(\vec{x})$, it is clear that we must continue these formulae to Minkowski space-time in order to make them physically meaningful. Thermal equilibrium having been assumed, there is no $x_{0}$ dependence anywhere to deal with. The only continuation needed is in the gauge potential

$$
A_{0}^{E}(\vec{x}) \rightarrow i A_{0}(\vec{x})
$$

and correspondingly in the background electric field

$$
\overrightarrow{\mathcal{E}}=-\vec{\nabla} A_{0}^{E} \rightarrow i \vec{E}=-i \vec{\nabla} A_{0}
$$

Making the change (3.2) wherever $\overrightarrow{\mathcal{E}}$ appears in the coefficients in eqs. (1.2)(1.4) as well as the replacement $\cos n \beta A_{0}^{E} \rightarrow \cosh n \beta A_{0}$, eqs.(1.2)-(1.4) become Minkowski space-time statements. The latter are the central results of the present article, obtained by general arguments based on gauge invariance and Fourier analysis.

At this point examples may be helpful. Let us quote the following two thermal heat kernels from refs. [17, 19] where the detailed calculations can be found.

Continuing (2.8) to Minkowski space we have for a spinor field in 1 spatial dimension coupled to $A_{\mu}=\left(E x_{1}+\mu, 0\right)[19]$ :

$$
\operatorname{trh}^{(\beta)}(t \mid x, x)=\frac{E}{2 \pi \tan E t} \sum_{n=-\infty}^{\infty}(-)^{n} e^{-n^{2} \beta^{2} E / 4 \tan E t} e^{n \beta\left(E x_{1}+\mu\right)} .
$$

For a scalar field in $d$ spatial dimensions coupled to $A_{\mu}=\left(E x_{1}+\mu, \overrightarrow{0}\right)$ 
[17]:

$$
h^{(\beta)}(t \mid x, x)=(4 \pi t)^{-\frac{d-1}{2}} \frac{E}{4 \pi \sin E t} \sum_{n=-\infty}^{\infty} e^{-n^{2} \beta^{2} E / 4 \tan E t} e^{n \beta\left(E x_{1}+\mu\right)} .
$$

The background in eqs. (3.3), (3.4) is a uniform electric field in the $x_{1}$ direction. The vacuum $(n=0)$ contributions

$$
\begin{array}{ll}
\text { spinor : } & \operatorname{tr} h^{(\beta)}(t)_{\text {sea }}=\frac{E}{2 \pi \tan E t}, \\
\text { scalar }: & h^{(\beta)}(t)_{\text {sea }}=(4 \pi t)^{-\frac{d-1}{2}} \frac{E}{4 \pi \sin E t},
\end{array}
$$

to the thermal heat kernels above display the ubiquitous singularity at $t=0$ and, in addition, singularities at $t=q \pi / E$ with $q=1,2,3 \ldots$ One does not expect to find the latter singularities in a physical heat kernel. They are present here because the vacuum is unstable: the background electric field produces (at a temperature-independent rate which does not directly involve the background voltage $\left.A_{0} / e\right)$ pairs of real particles from the sea. This has been discussed by Schwinger [12] and by others (see e.g. the books [13]- [15]).

The plasma contributions in eqs. (3.3), (3.4) - the sum of all $n \neq 0$ terms - display all of the properties mentioned earlier. They are nonsingular at $t=0$ : the thermal plasma is UV-finite. They have no singularities for $t>0$ : pair production from the sea is temperature-independent. They vanish exponentially as $T \rightarrow 0$ : the plasma disappears. Most importantly, they depend explicitly on the gauge potential $A_{0}=E x_{1}+\mu$ in the way we expect them to.

Global studies of thermal fields coupled to a uniform background $E$ are given in refs. [20]-23]. These investigations do not find the $\cosh \left[m \beta\left(E x_{1}+\mu\right)\right]$ dependence in local plasma quantities. For large $x_{1}$ the factors $\cosh \left[m \beta\left(E x_{1}+\right.\right.$ $\mu)$ ] diverge . However, the meaning of this (apparent) divergence can be explained in very physical terms. It is the result of the background voltage function which is unbounded as $x_{1} \rightarrow \pm \infty$, this being of course, an idealization associated with a uniform electric field of infinite spatial extent. 


\section{Parallel Uniform Electric and Magnetic Fields}

To further illustrate the Fourier series (1.2)-(-1.4), we now discuss the problem of parallel uniform $\vec{E}$ and $\vec{B}$ fields coupled to a thermal scalar field. Parallel $\vec{E}$ and $\vec{B}$ exert mutually perpendicular electric and magnetic forces on individual charged particles. Mathematically this leads to a complete factorization of the electric and magnetic sectors. Global treatments of the spinor version of this problem can be found in refs. [21]-23]. The $T=0$ problem was solved by Schwinger long ago [12].

\subsection{Infinite space}

For the Euclidean gauge potential $A_{\mu}^{E}(\vec{x})=\left(\mathcal{E} x_{1}+c_{0}, 0,0, B x_{2}+c_{3}\right)$ corresponding to a uniform background magnetic field $\vec{B}=(B, 0,0)$ parallel to the Euclidean electric field $\overrightarrow{\mathcal{E}}=(\mathcal{E}, 0,0)$, the mode equation (2.2) separates. With $p=\left(n, n^{\prime}, k\right)$ and modes

$$
\varphi_{m p}(\vec{x})=\frac{1}{\sqrt{2 \pi}} e^{i k x_{3}} \psi_{m n}\left(x_{1}\right) \psi_{k n^{\prime}}\left(x_{2}\right)
$$

eq. (2.2) separates into

$$
\left[-\partial_{1}^{2}+\mathcal{E}^{2}\left(x_{1}+c_{0} / \mathcal{E}-2 \pi m / \beta \mathcal{E}\right)^{2}\right] \psi_{m n}\left(x_{1}\right)=2 \mathcal{E}(n+1 / 2) \psi_{m n}\left(x_{1}\right)
$$

and

$$
\left[-\partial_{2}^{2}+B^{2}\left(x_{2}+c_{3} / B-k / B\right)^{2}\right] \psi_{k n^{\prime}}\left(x_{2}\right)=2 B\left(n^{\prime}+1 / 2\right) \psi_{k n^{\prime}}\left(x_{2}\right),
$$

where $n, n^{\prime}=0,1,2, \ldots$ and

$$
\lambda_{m p}^{2}=2 \mathcal{E}(n+1 / 2)+2 B\left(n^{\prime}+1 / 2\right)
$$

A peculiarity of this spectrum is its lack of dependence on $m$ and $k$. This degeneracy does complicate the calculation of global quantities but not, as we shall see, of local functions. One knows the eigenvalues and eigenfunctions 
in eqs. (4.2), (4.3) since they are both harmonic oscillator (HO) equations in $d=1$. Hence the corresponding eigenfunctions are

$$
\psi_{m n}\left(x_{1}\right)=2^{-n / 2} \frac{1}{\sqrt{n !}}\left(\frac{\mathcal{E}}{\pi}\right)^{\frac{1}{4}} e^{-\frac{1}{2} \mathcal{E} x_{m}^{2}} H_{n}\left(\sqrt{\mathcal{E}} x_{m}\right)
$$

with

$$
x_{m} \equiv x_{1}+\frac{c_{0}}{\mathcal{E}}-\frac{2 \pi m}{\beta \mathcal{E}}, \quad m=0,1,2, \cdots
$$

and

$$
\psi_{k n^{\prime}}\left(x_{2}\right)=2^{-n^{\prime} / 2} \frac{1}{\sqrt{n^{\prime} !}}\left(\frac{B}{\pi}\right)^{1 / 4} e^{-\frac{1}{2} B x_{k}^{2}} H_{n^{\prime}}\left(\sqrt{B} x_{k}\right)
$$

with

$$
x_{k}=x_{2}+\left(c_{3}-k\right) / B, \quad n^{\prime}=0,1,2, \ldots
$$

Here $H_{n}^{\prime}(z)$ are Hermite polynomials satisfying $H_{n^{\prime}}^{\prime \prime}-2 z H_{n^{\prime}}^{\prime}+2 n H_{n^{\prime}}=0$. The (diagonal) heat kernel constructed from the modes (4.5) is respectivley (see [17] for details)

$$
h_{1}^{(\beta)}(t \mid x, x)=\frac{\mathcal{E}}{4 \pi \sin \mathcal{E} t} \sum_{n=-\infty}^{\infty} e^{-n^{2} \beta^{2} \mathcal{E} / 4 \tan \mathcal{E} t} e^{n \beta\left(\mathcal{E} x_{1}+\mu\right)}
$$

The (off diagonal) heat kernel constructed from the modes (4.7) is

$$
h_{2}(t \mid x, y)=\left[\frac{B}{2 \pi \sinh 2 B t}\right]^{\frac{1}{2}} \frac{1}{2 \pi} \int_{-\infty}^{\infty} d k e^{-\frac{1}{2} B\left(x_{k}-y_{k}\right)^{2} \operatorname{coth} 2 B t} e^{-B x_{k} y_{k} \tanh B t},
$$

where again the details of the calculation parallel those in ref. [17].

Putting things together, the diagonal heat kernel for parallel electric and magnetic fields can now be written down (using $\mathcal{E}=i E, c_{0}=i \mu$ to continue to Minkowski space-time):

$$
h^{(\beta)}(t \mid x, x)=\frac{B}{4 \pi \sinh B t} \frac{E}{4 \pi \sin E t} \sum_{n=-\infty}^{\infty} e^{-n^{2} \beta^{2} E / 4 \tan E t} \times e^{n \beta\left(E x_{1}+\mu\right)}
$$

where the integration over $k$ has eliminated all dependence on the spatial coordinate $x_{2}$ and on the constant $c_{3}$ from the diagonal local heat kernel. 


\subsection{Arbitrary $B_{1}\left(x_{2}\right)$}

The factorization of the electric and magnetic sectors for parallel $\overrightarrow{\mathcal{E}}$ and $\vec{B}$ fields can be further exploited. Let us replace the potential $A_{3}=B x_{2}+c_{3}$ above by an arbitrary function $A_{3}\left(x_{2}\right)$ of $x_{2}$. Then the background magnetic field $B_{1}=\partial_{2} A_{3}$ has an arbitrary dependence on $x_{2}$. The modes (4.1) still factorize, and eq. (4.3) is replaced by

$$
\left[-\partial_{2}^{2}+\left(A_{3}\left(x_{2}\right)-k\right)^{2}\right] \Psi_{k n^{\prime}}\left(x_{2}\right)=w_{k n^{\prime}}^{2} \Psi_{k n^{\prime}}\left(x_{2}\right) .
$$

Now $\lambda_{m p}^{2}=2 \mathcal{E}(n+1 / 2)+w_{k n^{\prime}}^{2}$, with the (unknown) eigenfunctions $\Psi_{k n^{\prime}}\left(x_{2}\right)$ and spectrum $\left\{w_{k n^{\prime}}^{2}\right\}$ determined by the mode equation just above. The heat kernel $(4.10)$ is replaced by

$$
h^{(\beta)}(t \mid x, x)=h_{2}^{(\beta)}\left(t \mid x_{2}, x_{2}\right)[E \text { dependent factor in eq.4.10)] }
$$

with

$$
h_{2}\left(t \mid x_{2}, x_{2}\right)=\int d k \sum_{n^{\prime}} e^{-t w_{k n^{\prime}}^{2}}\left|\Psi_{k n^{\prime}}\left(x_{2}\right)\right|^{2}
$$

in place of $h_{2}=B / 4 \pi \sinh B t$. Obviously the Fourier series structure of the heat kernel is preserved, even for arbitrary $B_{1}\left(x_{2}\right)$.

\subsection{Cylindrical space}

If spatial direction $x_{3}$ were compact - say $0 \leq x_{3} \leq L$ - the conjugate momentum $k$ would be discrete: $k=r(2 \pi / L)$ with $r=0, \pm 1, \pm 2, \ldots$. Then the integral (4.9) would become a sum over $r$, exactly the same mode sum which leads to the Euclidean version of the electric field factor in eq. (4.10), with $L$ and $i B$ in place of $\beta$ and $E$. Thus the above compactification of $x_{3}$ leads to the thermal heat kernel

$$
\begin{aligned}
h^{(\beta)}(t \mid x, x)= & \frac{B}{4 \pi \sinh B t} \sum_{r} e^{-r^{2} L^{2} B / \tanh B t} \times e^{i r L\left(B x_{2}+c_{3}\right)} \\
& \times \frac{E}{4 \pi \sin E t} \sum_{n} e^{-n^{2} \beta^{2} E / \tan E t} \times e^{n \beta\left(E x_{1}+\mu\right)},
\end{aligned}
$$


eq. (4.10) being the $L=\infty$ limit of this. By compactifying the spatial axis $x_{3}$, the gauge potential $A_{3}=x_{2} B+c_{3}$ has turned into a compact local variable $O \leq L A_{3} \leq 2 \pi$, very much as the compactification of Euclidean time leads to the compactification of $\beta A_{0}^{E}$. This has nothing to do with the electric field and remains true at zero temperature and $E=0$.

Interesting mathematical physics is associated with the compactification of $L A_{3}$; however, this lies beyond the scope of the present paper. We mention some early literature (see e.g. refs. [24, 25]) which investigates the effect of $x_{3}$ compactification on a $T=0$ QFT.

\section{$5 \quad$ Perpendicular Electric and Magnetic Fields}

Finally we work through the quite different problem of perpendicular background $\vec{E}$ and $\vec{B}$-fields. For such a background the magnetic force on moving charges has a component in the direction of the electrostatic force on the same charge. This couples the electric and magnetic sectors, eliminating the factorization observed for $\vec{E} \| \vec{B}$ in the preceeding section. See refs. 21] -23 for the spinor version of this system (treated globally) and Schwinger 12 for the $T=0$ problem.

Choosing the Euclidean gauge potential $A_{\mu}^{E}(x)=\left(\mathcal{E} x_{1}+C_{0}, 0, B x_{1}+C_{2}, 0\right)$ corresponding to background (Euclidean) electric and magnetic fields $\overrightarrow{\mathcal{E}}=$ $(\mathcal{E}, 0,0)$ and $\vec{B}=(0,0, B)$, respectively, the mode operator in eq. (2.2) is

$$
\begin{aligned}
-D^{2} & =\left(\mathcal{E} x_{1}+C_{0}-\frac{2 \pi m}{\beta}\right)^{2}-\partial_{1}^{2}+\left(-k_{2}+B x_{1}+C_{2}\right)^{2}+k_{3}^{2} \\
& =-\partial_{1}^{2}+w^{2}\left(x_{1}-u\right)^{2}+v^{2}+k_{3}^{2}
\end{aligned}
$$

where

$$
\begin{gathered}
w^{2} u=\mathcal{E}\left(\frac{2 \pi m}{\beta}-C_{0}\right)+B\left(k_{2}-C_{2}\right), \\
w^{2} v^{2}=\left[B\left(\frac{2 \pi m}{\beta}-C_{0}\right)-\mathcal{E}\left(k_{2}-C_{2}\right)\right]^{2},
\end{gathered}
$$


and $w^{2}=\mathcal{E}^{2}+B^{2}$. We have included the constant term in $A_{2}=B x_{1}+C_{2}$ even though we know that $C_{2}$ cannot appear in physical quantities, for it is of some interest to see how the mathematics eliminates $C_{2}$. In eq. (5.1) we have assumed the modes (2.2) (with $p=\left(n, k_{2}, k_{3}\right)$ ) to be of the factorized form

$$
\varphi_{m p}(\vec{x})=\frac{1}{2 \pi} e^{i\left(k_{2} x_{2}+k_{3} x_{3}\right)} \varphi_{m n k_{2}}\left(x_{1}\right) .
$$

The eigenvalues $\lambda_{m p}^{2}$ of the operator (5.1) are then given by

$$
\lambda_{m p}^{2}=2 w\left(n+\frac{1}{2}\right)+k_{3}^{2}+v^{2}
$$

with $H O$ eigenfunctions $\varphi_{m n k_{2}}\left(x_{1}\right)=\varphi_{n}\left(x_{1}-u\right)$, where $\varphi_{n}$ is given by

$$
\varphi_{n}(x)=2^{-n / 2} \frac{1}{\sqrt{n !}}\left(\frac{w}{\pi}\right)^{\frac{1}{4}} e^{-\frac{1}{2} w x^{2}} H_{n}(\sqrt{w} x),
$$

The result of the calculation of the Euclidean thermal space-time heat kernel (2.3) is expedited by eq. (4.9) with the substitution $B \rightarrow w$. For the diagonal heat kernel one finds

$$
\begin{aligned}
h^{(\beta)}(t \mid x, x)= & \frac{1}{\beta} \sum_{m} \frac{1}{4 \pi^{2}} \int d k_{2} d k_{3} e^{-t k_{3}^{2}} e^{-t v^{2}} \\
& \times\left[\frac{w}{2 \pi \sinh 2 w t}\right]^{1 / 2} e^{-w \tanh w t\left(x_{1}-u\right)^{2}}
\end{aligned}
$$

with $u$ and $v^{2}$ given by eqs (5.2), (5.3). The change of variable (which eliminates $C_{2}$ )

$$
w v=\mathcal{E}\left(k_{2}-C_{2}\right)-B\left(\frac{2 \pi m}{\beta}-C_{0}\right)
$$

leads to

$$
\begin{aligned}
h^{(\beta)}(t \mid x, x)= & (4 \pi t)^{-1 / 2}\left[\frac{w}{2 \pi \sinh 2 w t}\right]^{1 / 2} \frac{w}{\mathcal{E}} \int_{-\infty}^{\infty} d v e^{-t v^{2}} \\
& \times \frac{1}{\beta} \sum_{m=-\infty}^{\infty} e^{-w \tanh w t\left(x_{1}-u\right)^{2}}
\end{aligned}
$$

with now

$$
u=\frac{1}{\mathcal{E}}\left(\frac{2 \pi m}{\beta}-C_{0}\right)+\frac{B}{w \mathcal{E}} v
$$


The Matsubara sum is done with the help of a well-known theta function identity

$$
\begin{aligned}
& \frac{1}{\beta} \sum_{m=-\infty}^{\infty} e^{-w \tanh w t\left(x_{1}-u\right)^{2}}=\mathcal{E}\left[\frac{1}{4 \pi w \tanh w t}\right]^{1 / 2} \\
& \times \sum_{n=-\infty}^{\infty} e^{-n^{2} \mathcal{E} \beta^{2} / 4 w \tanh w t} e^{-i n \beta\left(\mathcal{E} x_{1}+C_{0}\right)} e^{i n \beta B v / w}
\end{aligned}
$$

Finally we employ

$$
\frac{1}{2 \pi} \int_{-\infty}^{\infty} d v e^{-t v^{2}} e^{i n \beta B v / w}=(4 \pi t)^{-1 / 2} e^{-n^{2} \beta^{2} B^{2} / 4 w^{2} t}
$$

to write the heat kernel (5.8) in the form

$$
\begin{aligned}
h^{(\beta)}(t \mid x, x) & =(4 \pi t)^{-1} \frac{w}{4 \pi \sinh w t} \\
& \times \sum_{m=-\infty}^{\infty} e^{-m^{2} \beta^{2} \mathcal{E} / 4 w \tanh w t} e^{-m^{2} \beta^{2} B^{2} / 4 w^{2} t} e^{-i m \beta\left(\mathcal{E} x_{1}+C_{0}\right)}
\end{aligned}
$$

which has the expected form (2.3) with nonalternating sign. Moreover, for $B \rightarrow 0$ or $\mathcal{E} \rightarrow 0$ this heat kernel has the correct limits.

\section{Conclusion}

Our main result is that for a thermal charged matter field coupled to a static electromagnetic background gauge potential $A_{\mu}(\vec{x})$ the thermal plasma - but not the virtual sea - feels locally the potential $A_{0}(\vec{x})$ in addition to the gaugeinvariant electric and magnetic fields $\vec{E}=-\vec{\nabla} A_{0}$ and $\vec{B}=\vec{\nabla} \times \vec{A}$. This was discovered in the context of specific calculations [17, 19] involving a constant background $\vec{E}$, with $\vec{B}=0$. Here we have explained the underlying general principles and generalized the discussion to an arbitrary static potential $A_{0}(\vec{x})$ (and hence also an arbitrary static electric field) and an arbitrary static magnetic field $\vec{B}(\vec{x})$. For reasons of gauge invariance the Euclidean gauge potential $A_{0}^{E}(\vec{x})$ is a local compact variable in any local function describing the many-body quantum system. This function therefore has a Fourier cosine series expansion in $\beta A_{0}^{E}(\vec{x})$, in which the term independent of $A_{0}^{E}$ represents 
the virtual sea contribution. Continued to Minkowski spacetime, this series becomes a hyperbolic cosine expansion in the Minkowski potential $\beta A_{0}(\vec{x})$, displaying the chemical-potential-like role of a constant background voltage for the charged thermal field.

In sections 4 and 5 we then extended our previous explicit scalar field

calculation with $\vec{B}=0$ to the two most important backgrounds with uniform $\vec{E}$ and $\vec{B}$ : namely $\vec{E} \| \vec{B}$ and $\vec{E} \perp \vec{B}$. Completely explicit Fourier series were obtained for the thermal heat kernels of these systems, thereby providing additional, more complex examples of the general theory. For brevity we have not included (although one easily could) effective Lagrangians, energy momentum tensors and the like in these examples. Our goal has been to provide new insight into the local aspects of thermal matter fields coupled to static electromagnetic backgrounds. We hope to present more complete results for interesting systems at a later time.

\section{References}

[1] N. Batakis and G. Lazarides, Phys. Rev. D18 (1978) 4710.

[2] I. Affleck, Nucl. Phys. B162 (1980) 461; 171 (1980) 420.

[3] A. M. Polyakov, Phys. Lett. 72B (1978) 477.

[4] E. Gava, R. Jengo, and C. Omero, Nucl. Phys. B170[FS1] (1980) 445.

[5] D. J. Gross, R. D. Pisarski, and L. G. Yaffe, Rev. Mod. Phys. 53 (1981) 43.

[6] N. Weiss, Phys. Rev. D24 (1981) 475; 25 (1982) 2667.

[7] A. Actor, Phys. Rev. D27 (1983) 2548; Ann. Phys. (N.Y.) 159 (1985).

[8] T. Matsubara, Prog. Theor. Phys. 14 (1955) 351. 
[9] A. Das, Finite Temerpature Field Theory, World Scientific, Singapore, 1997.

[10] P. D. Morley and M. B. Kislinger, Phys. Rep. 51 (1979) 63.

[11] J. I. Kapusta, Phys. Rev. D24 (1981) 426.

[12] J. Schwinger, Phys. Rev. 82 (1951) 664.

[13] W. Dittrich and M. Reuter, Lecture Notes in Physics: Effective Lagrangians in Quantum Electrodynamics, vol. 220, Springer, Berlin, 1984.

[14] W. Greiner, B. Mueller, and J. Rafelski, Quantum Electrodynamics of Strong Fields, Springer, Berlin 1985.

[15] E. S. Fradkin, D. M. Gitman, and Sh. M. Shvartsman, Quantum Electrodynamics with Unstable Vacuum, Springer, Berlin, 1991.

[16] Y. Kluger, J.M. Eisenberg, B. Svetilsky, F. Cooper and E. Mottola, Phys. Rev. Lett. 67 (1991) 2427; Phys. Rev. D45 (1992) 4659.

[17] A. Actor, K. Rothe, and F. Scholtz, J. Phys. A: Math. Gen. 32 (1999) 7463.

[18] D. M. McAvity and H. Osborn, Class. Quantum Grav. 8 (1991) 603.

[19] A. Actor, K. Rothe, and F. Scholtz, Phys. Rev. D60 (1999) 105034.

[20] A. Cabo, Fortschr. der Physik 29 (1981) 495.

[21] P. H. Cox, W. S. Hellman, and A. Yildiz, Ann. Phys. (N.Y.) 154 (1984) 211.

[22] M. Loewe and J. C. Rojas, Phys. Rev. D46 (1992) 2689.

[23] P. Elmfors and Bo-S. Skagerstam, Phys. Lett. B348 (1995) 141. 
[24] D. J. Toms, Phys. Rev. D21 (1980) 928, 2805; Phys. Lett. 77A (1980) 303.

[25] L. H. Ford, Phys. Rev. D21 (1980) 933; D22 (1980) 330, 3003.

[26] H. Boschi-Filho, C.P. Natividade and C. Farina, Phys. Rev. D45 (1992) 586.

[27] E. Abdalla, M.C.B. Abdalla and K.D. Rothe, Non Perturbative Methods in 2 Dimensional Quantum Field Theory, World Scientific, Singapore, 1991. 